ARTICLE

DOI: $10.1038 / s 41467-018-07372-x$

\title{
Control of spin-wave transmission by a programmable domain wall
}

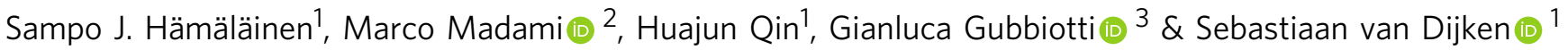

Active manipulation of spin waves is essential for the development of magnon-based technologies. Here, we demonstrate programmable spin-wave filtering by resetting the spin structure of pinned $90^{\circ}$ Néel domain walls in a continuous CoFeB film with abrupt rotations of uniaxial magnetic anisotropy. Using micro-focused Brillouin light scattering and micromagnetic simulations, we show that broad $90^{\circ}$ head-to-head or tail-to-tail magnetic domain walls are transparent to spin waves over a broad frequency range. In contrast, magnetic switching to a $90^{\circ}$ head-to-tail configuration produces much narrower and strongly reflecting domain walls at the same pinning locations. Based on these results, we propose a magnetic spin-wave valve with two parallel domain walls. Switching the spin-wave valve from an open to a closed state changes the transmission of spin waves from nearly 100 to $0 \%$. Active control over spin-wave transport through programmable domain walls could be utilized in magnonic logic devices or non-volatile memory elements.

\footnotetext{
${ }^{1}$ NanoSpin, Department of Applied Physics, Aalto University School of Science, P.O. Box 15100, FI-00076 Aalto, Finland. ${ }^{2}$ Dipartimento di Fisica e Geologia, Università di Perugia, 06123 Perugia, Italy. ${ }^{3}$ Istituto Officina dei Materiali del CNR (CNR-IOM), Sede Secondaria di Perugia, c/o Dipartimento di Fisica e Geologia, Università di Perugia, 06123 Perugia, Italy. Correspondence and requests for materials should be addressed to

S.v.D. (email: sebastiaan.van.dijken@aalto.fi)
} 
W ave-like computing based on spin waves has generated interest as a potential low-power and parallel computing alternative for conventional CMOS technologies $^{1}$. High group velocities ${ }^{2}$, long decay lengths ${ }^{3}$, and the ability to tailor the wavelength of spin waves down to the nanoscale ${ }^{4-6}$ offer fascinating prospects for magnonics. Logic devices based on a Mach-Zehnder spin-wave interferometer have been proposed and realized ${ }^{7-10}$. In this geometry, spin waves are launched into the interferometer branches, and their phase or amplitude is controlled by an Oersted field ${ }^{7-9}$ or spin-wave current from a third magnetic terminal ${ }^{10}$. Interference of spin-wave signals after manipulation determines the logic output. Other logic concepts exploit reprogrammable magnonic crystals ${ }^{11,12}$. Here, spin-wave transmission is controlled actively by a spatial modulation of magnetic properties using, for instance, electric currents ${ }^{13}$ or optical pulses ${ }^{14}$. Besides these dynamic approaches, field-induced toggling between ferromagnetic and antiferromagnetic states in magnetic stripe arrays has been shown to modify spin-wave transport in a non-volatile way ${ }^{15,16}$.

Non-collinear spin structures, such as magnetic domain walls, can also be exploited to control the amplitude or phase of spin waves ${ }^{17,18}$. It has been analytically calculated that spin-wave transport through an infinitely extended one-dimensional Bloch wall induces a finite phase shift without reflection ${ }^{19}$. In contrast, interactions between spin waves and $180^{\circ}$ Néel walls or magnetic domain walls in confined geometries are more complex ${ }^{20}$. Dynamic stray fields in such domain walls reduce the transmission of spin waves if their wavelength exceeds the wall width ${ }^{21,22}$. Additionally, domain-wall resonances limit the transmission of spin waves at specific frequencies ${ }^{23-26}$. This effect, known as resonant reflection, relates strongly to the spatial inhomogeneity of the effective magnetic field inside the wall. Thus, because of dynamic stray fields and resonance modes, narrow domain walls reflect spin waves more than broad walls. In many studies, the interaction between spin waves and magnetic domain walls is investigated as a new method to drive walls into motion ${ }^{22-29}$. It has been shown that the direction and velocity of wall motion depend strongly on the spin-wave transmission coefficient. For coefficients close to unity, transfer of angular momentum causes domain walls to move against the spin waves ${ }^{27,28}$. In contrast, strong spin-wave reflection induces forward domain-wall motion $^{22-26}$.
In this paper, we propose the use of programmable domain walls for active spin-wave manipulation (Fig. 1a). Periodic $90^{\circ}$ rotations of uniaxial magnetic anisotropy firmly pin Néel-type walls in our system, preventing their motion under the action of propagating spin waves. Because of pinning, a magnetic field can reversibly transform a narrow $90^{\circ}$ head-to-tail domain wall into a broad $90^{\circ}$ head-to-head or tail-to-tail wall. Using phase-resolved micro-focused Brillouin light scattering ( $\mu$-BLS) and micromagnetic simulations, we show that broad domain walls are transparent for spin waves over a wide frequency range. In contrast, spin waves are resonantly reflected by narrow domain walls.

\section{Results}

Experimental realization. Interactions of spin-waves with $90^{\circ}$ Néel walls have been imaged in square-shaped ferromagnetic microstructures with a Landau domain state ${ }^{30,31}$. The head-to-tail domain walls in these experiments act as barriers for spin-wave transport. To realize active switching between such narrow domain walls and broader $90^{\circ}$ walls with a head-to-head or tailto-tail structure, we couple a $50 \mathrm{~nm}$ thick ferromagnetic $\mathrm{CoFeB}$ film to a ferroelectric $\mathrm{BaTiO}_{3}$ substrate with regular ferroelastic stripe domains (Fig. 1b). The combination of strain transfer and inverse magnetostriction in this bilayer system causes imprinting of magnetic stripe domains in the ferromagnetic layer ${ }^{32,33}$. The magnetic anisotropy of the $\mathrm{CoFeB}$ film is uniaxial, reflecting the tetragonal symmetry of the underlying ferroelectric crystal, and the anisotropy axis rotates abruptly by $90^{\circ}$ between domains. In a previous study, we showed that excitation of this system by an uniform microwave magnetic field results in the formation of standing spin waves within the domains and spin-wave emission from the anisotropy boundaries ${ }^{34}$. Here, we focus on spin-wave transport through $90^{\circ}$ Néel walls. Importantly, strong pinning of straight magnetic domain walls at the anisotropy boundaries enables switching between head-to-tail and head-to-head/tail-totail walls in the CoFeB film. We use an external in-plane magnetic field to toggle between these non-volatile magnetization states. If the field is applied along the domain boundaries and turned off, the magnetization aligns in an alternating head-to-head and tailto-tail configuration (Fig. 1c, top). The same process after rotating the in-plane field by $90^{\circ}$ initializes head-to-tail domain walls

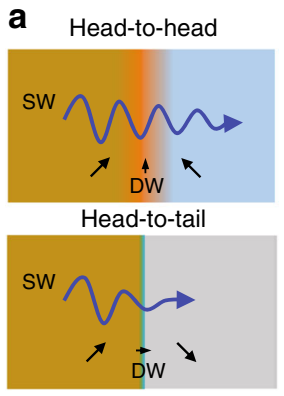

b

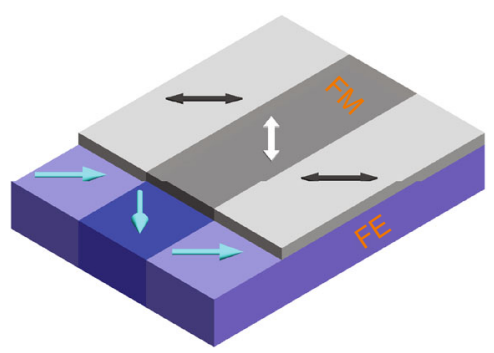

C

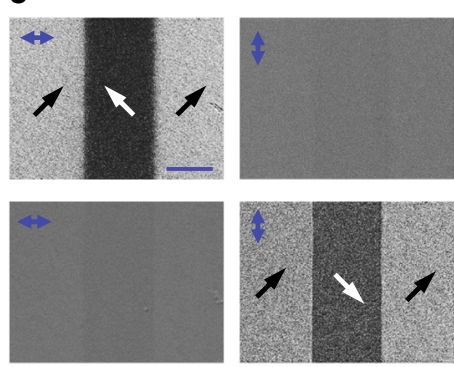

Fig. 1 Spin-wave control via programming of pinned magnetic domain walls. a Schematic of spin-wave (SW) transmission through a pinned $90^{\circ}$ magnetic domain wall (DW) with a head-to-head and head-to-tail structure. The pinned domain wall in the ferromagnetic film separates stripe domains with uniaxial magnetic anisotropy. Because the anisotropy axis rotates by $90^{\circ}$ at the domain boundary, a magnetic field along the boundary initializes a head-to-head (top) or tail-to-tail wall. These domain walls are broad and transparent to spin waves. Application of a magnetic field perpendicular to the boundary results in the formation of a much narrower head-to-tail domain wall (bottom). Spin waves are mostly reflected by this wall. Reversible magnetic switching of the domain-wall structure thus actively alters the transmission of spin waves. b Illustration of the experimental $\mathrm{CoFeB} / \mathrm{BaTiO} \mathrm{O}_{3}$ bilayer system. The domain pattern of the ferroelectric (FE) substrate is imprinted into the ferromagnetic (FM) film via strain transfer and inverse magnetostriction. Blue arrows and double-headed black and white arrows indicate the direction of ferroelectric polarization and the orientation of uniaxial magnetic anisotropy. c Magnetooptical Kerr microscopy images of the magnetization configuration in a $\mathrm{CoFeB} / \mathrm{BaTiO}_{3}$ bilayer after the application of a magnetic field along the domain boundaries (top) and perpendicular to the domain boundaries (bottom). The left and right images are taken with the magneto-optical contrast axis along the horizontal and vertical direction, respectively (blue arrows). The blue scale bar corresponds to $10 \mu \mathrm{m}$ 
(Fig. 1c, bottom). Both walls are of the $90^{\circ}$ Néel type in zero magnetic field, but their widths differ significantly ${ }^{35}$. Scanning electron microscopy with polarization analysis (SEMPA), X-ray photoemission electron microscopy (XPEEM), and micromagnetic simulations on $\mathrm{CoFeB} / \mathrm{BaTiO}{ }_{3}$ and related systems have previously shown that the width $(\delta)$ of $90^{\circ}$ head-to-head/tail-totail domain walls increases sharply with CoFeB film thickness $(t)$ because of their large magnetostatic energy $(\delta \propto t)^{36-38}$. In contrast, the width of $90^{\circ}$ head-to-tail domain walls is almost entirely determined by a competition between ferromagnetic exchange and the strength of magnetic anisotropy and, thus, varies much less with $t^{36}$. Consequently, the difference in domain wall width is more pronounced in thick ferromagnetic films. In our experiments, we focus on 50-nm-thick CoFeB because it combines full imprinting of magnetic stripe domains (Fig. 1b, c) with the ability to reprogram the domain wall width from $\sim 50 \mathrm{~nm}$ (head-to-tail wall) to $1.6 \mu \mathrm{m}$ (head-to-head and tail-to-tail wall). Details on sample preparation are given in the Methods section.

Brillouin light scattering experiments. Dispersion relations of spin waves depend on the angle between the wave vector and direction of magnetization. In most spin-wave experiments, a magnetic bias field is used to saturate the magnetization of a sample either parallel or perpendicular to the wave vector. Because of strain-induced uniaxial magnetic anisotropy in the $\mathrm{CoFeB}$ film of our bilayer, the magnetization is uniform within the stripe domains in zero magnetic field. This enabled us to perform measurements without bias field and to study spin-wave transport through $90^{\circ}$ Néel walls. In the remanent state, the $\mathrm{CoFeB}$ magnetization aligns along the uniaxial magnetic anisotropy axes of the stripe domains, i.e., at an angle of $45^{\circ}$ with respect to the domain walls (Fig. 1c). The type of magnetic domain wall was set before spin-wave characterization by either applying a parallel or perpendicular magnetic field.

We employed $\mu$-BLS to measure spin-wave transmission through pinned magnetic domain walls. For the excitation of spin waves, we patterned 500-nm-wide microwave antennas on top of our sample using electron-beam lithography. The antennas are separated from the $\mathrm{CoFeB}$ film by an insulating $\mathrm{TaO}_{x}$ layer, and they are aligned parallel to a nearby domain wall (Fig. 2a, top). The microwave antennas excite spin waves over a broad range of wave vectors (the shortest wavelength is about $1 \mu \mathrm{m}$ ). In the experiments described below, the antenna edge and domainwall center are separated by $\sim 2 \mu \mathrm{m}$ (Fig. $2 \mathrm{a}$, bottom).

Figure $2 \mathrm{~b}$ shows $\mu$-BLS spectra measured at a fixed location 4.5 $\mu \mathrm{m}$ from the antenna edge (i.e., $2.5 \mu \mathrm{m}$ beyond the center of the pinned magnetic domain wall) and by scanning the $\mathrm{rf}$ excitation frequency in the range $5-15 \mathrm{GHz}$. The black and red curves depict BLS intensity data for a broad $90^{\circ}$ head-to-head and narrow $90^{\circ}$ head-to-tail domain wall, respectively. Two main spin-wave resonances are measured. The peaks measured at $\pm 12.6 \mathrm{GHz}$ are the same for both domain walls. We attribute these resonances to thermally excited perpendicular standing spin waves (PSSWs) in the CoFeB film because the peaks are recorded also when the rf-source is turned off and the dependence of their frequency on external magnetic field closely follows the calculated dispersion of the first-order PSSW mode (Supplementary Fig. 1). The other main resonances ranging from \pm 7.7 to $\pm 12.2 \mathrm{GHz}$ in the $\mu$-BLS spectra of Fig. $2 \mathrm{~b}$ illustrate the intensity of propagating spin waves after transmission through the magnetic domain wall. Since we maintained all experimental conditions, the BLS intensities for both magnetization configurations can be compared directly. The local $\mu$-BLS measurements indicate that spin waves are transmitted through the broad $90^{\circ}$ head-to-head domain wall and that their intensity is significantly suppressed by the narrow $90^{\circ}$ head-to-tail domain wall. The same conclusions can be drawn from phase-resolved $\mu$-BLS scans across the magnetic domain walls (Fig. 2c). In these phase-resolved measurements, $\mu$-BLS line scans were performed along the orange arrow in Fig. 2a. While the signal intensity drops significantly behind the head-to-tail domain wall, we do not measure a similar suppression after we switch the magnetization to the head-to-head configuration.

Figure 3 shows $\mu$-BLS line scans (a,d) and 2D intensity scans (b, c, e, f), measured at two different excitation frequencies. The measurements illustrate the decay of spin-wave intensity from the antenna edge across the pinned domain wall at $9.85 \mathrm{GHz}(\mathrm{a}-\mathrm{c})$ and $10.85 \mathrm{GHz}(\mathrm{d}-\mathrm{f})$. Line scans recorded at two other frequencies are shown in Supplementary Fig. 2. The data corroborate that initialization of a narrow $90^{\circ}$ head-to-tail domain wall at $x=0 \mu \mathrm{m}$ causes strong spin-wave reflection (red curves in Fig. 3a, d and 2D intensity scans in Fig. 3b, e). The BLS intensity after the pinned head-to-tail domain wall is almost
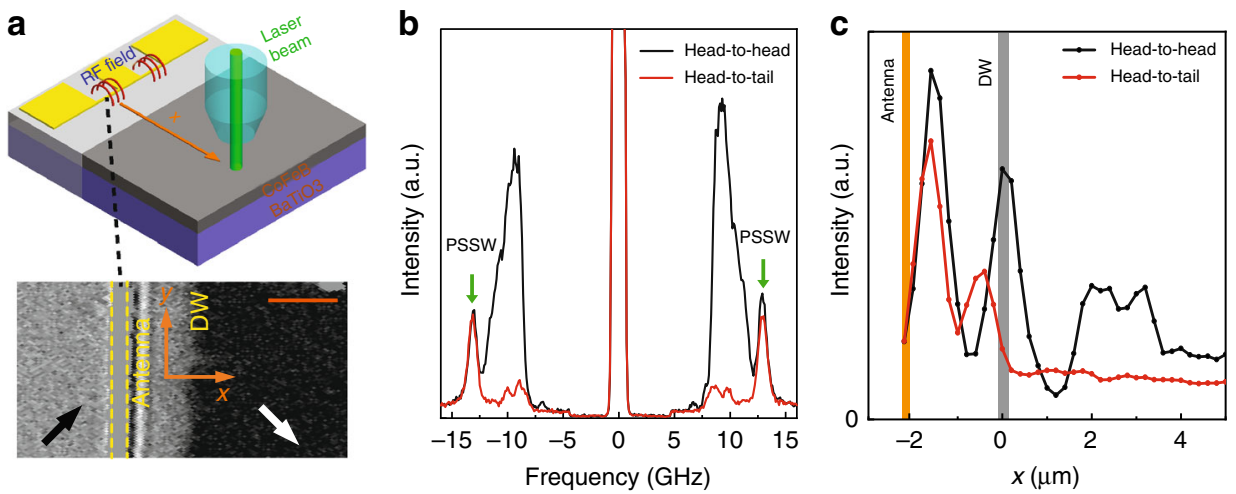

Fig. 2 Brillouin light scattering of spin-wave transmission. a Schematic and magneto-optical Kerr microscopy image of the $\mu$-BLS measurement geometry. A microwave antenna on top of the CoFeB film excites propagating spin waves. Transmission of these spin waves through a nearby head-to-head or head-totail domain wall is recorded by scanning the $\mu$-BLS laser beam across the wall (orange arrow in top panel). The orange scale bar in the bottom panel corresponds to $2 \mu \mathrm{m}$. $\mathbf{b} \mu$-BLS spectra measured at a fixed position, $4.5 \mu \mathrm{m}$ from the antenna, and by scanning the rf excitation frequency in the range 5 - 15 $\mathrm{GHz}$. Dissimilar transmission of propagating spin waves through the broad $90^{\circ}$ head-to-head and narrow $90^{\circ}$ head-to-tail domain wall is illustrated by the intensity peaks that range from \pm 7.7 to $\pm 12.2 \mathrm{GHz}$. The peak recorded at $12.6 \mathrm{GHz}$ on both the Stokes and anti-Stokes side of the spectra indicates thermal excitation of a PSSW mode in the CoFeB film. $\mathbf{c}$ Phase-resolved $\mu$-BLS scans across the pinned domain wall for head-to-head and head-to-tail magnetization configurations. The excitation frequency in this measurement is $10.85 \mathrm{GHz}$ 
a

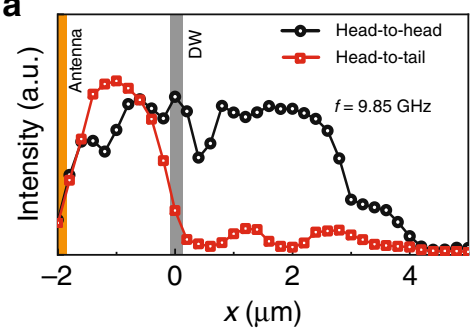

d

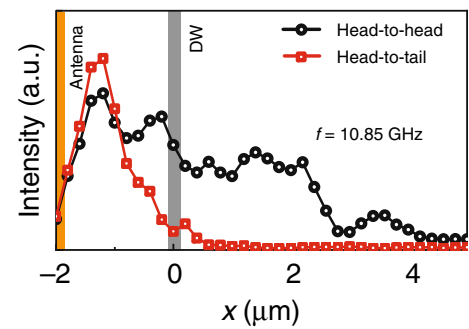

b

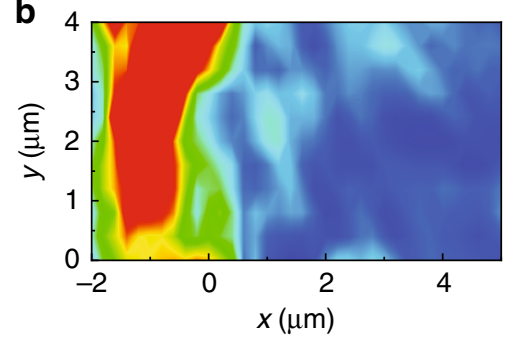

e

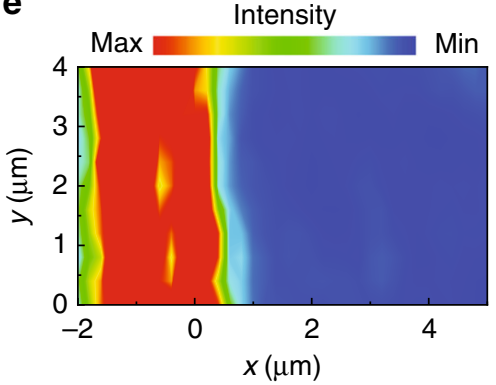

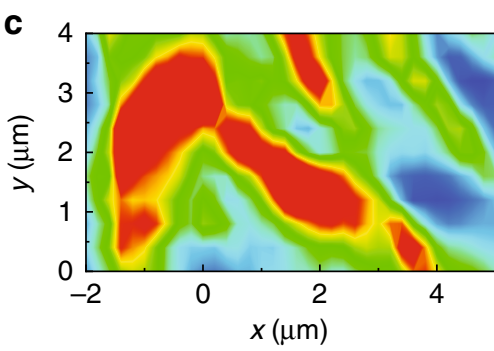

f
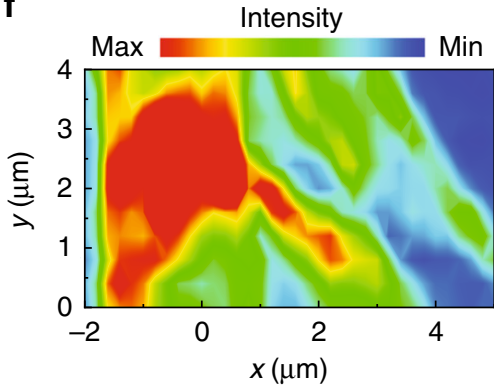

Fig. 3 Frequency and spatial dependence of spin-wave transmission. Line scans and areal maps of BLS intensity recorded from the antenna edge across the pinned domain wall at excitation frequencies of $9.85 \mathrm{GHz}(\mathbf{a}-\mathbf{c})$ and $10.85 \mathrm{GHz}$ (d-f). The areal maps in $\mathbf{b}$, e are recorded after the magnetization configuration is set to a head-to-tail domain wall. The maps in $\mathbf{c}$, $\mathbf{f}$ show BLS intensity data for a head-to-head domain wall

completely suppressed to the noise level at $10.85 \mathrm{GHz}$, whereas a small signal is measured at $9.85 \mathrm{GHz}$. In this magnetization configuration, the spin-wave signal already decays before the pinned domain wall. We attribute this effect to destructive interference of forward moving and reflected spin waves. The suppression is most pronounced at $10.85 \mathrm{GHz}$, providing additional proof that spin-wave reflection is particularly strong at this frequency. Switching the magnetization of the CoFeB film to a broad $90^{\circ}$ head-to-head domain wall drastically enhances the transmission of spin waves at all frequencies (black curves in Fig. 3a, $d$ and $2 \mathrm{D}$ intensity scans in Fig. 3c, f). In the 2D scans, we observe spatial non-uniformities in the spin-wave intensity. Similar BLS-intensity variations are also measured if we saturate the magnetization by an external magnetic field or if we characterize spin-wave transport using another antenna with no domain wall in its vicinity (Supplementary Fig. 3). The nonuniformities are therefore not caused by the domain wall, but rather by the granular structure of the $\mathrm{CoFeB}$ film, as illustrated by optical and atomic-force microscopy images in Supplementary Fig. 4.

Micromagnetic simulations. We performed micromagnetic simulations in $\operatorname{MuMax}^{39}$ to further analyze spin-wave transmission trough $90^{\circ}$ Néel walls. In the simulations, we considered two $20-\mu \mathrm{m}$-wide stripe domains. We used two-dimensional periodic boundary conditions and added a $1 \mu \mathrm{m}$ wide region with higher damping parameter at the edges of the simulation area to prevent spin-wave interference. We ensured that results for this geometry are identical to those of larger area simulations without periodic boundary conditions along $x$ and regions of higher damping. The structure was discretized using finite-difference 2.4 $\mathrm{nm} \times 9.6 \mathrm{~nm} \times 12.5 \mathrm{~nm}$ cells. To mimic anisotropy modulations in the experimental system, we abruptly rotated the uniaxial anisotropy axis by $90^{\circ}$ at the domain boundary. Input parameters were derived from experiments. We extracted $M_{\mathrm{s}}=1.15 \times 10^{6} \mathrm{~A} /$ $\mathrm{m}$ from vibrating sample magnetometry and $K_{\mathrm{u}}=2.5 \times 10^{4} \mathrm{~J} / \mathrm{m}^{3}$ from BLS for our 50 -nm-thick $\mathrm{CoFeB}$ film on $\mathrm{BaTiO}_{3}$. Additionally, we used an exchange constant of $A_{\mathrm{ex}}=2.1 \times 10^{-11} \mathrm{~J} / \mathrm{m}$ and a damping parameter of $\alpha=0.005$. For these parameters, we simulated a domain wall width of $50 \mathrm{~nm}$ (head-to-tail wall) and $1.6 \mu \mathrm{m}$ (head-to-head and tail-to-tail wall). Spin waves were excited locally by an out-of-plane sinusoidal magnetic field at the center of one of the domains, i.e., $10 \mu \mathrm{m}$ from the pinned domain wall. More details on micromagnetic simulations are given in the Methods section.

Figure 4 summarizes simulation results for spin-wave transmission through a $90^{\circ}$ head-to-head (a, c) and $90^{\circ}$ head-to-tail (d, f) domain wall at a frequency of $10.85 \mathrm{GHz}$. In the head-to-head configuration, the effective magnetic field reduces gradually inside the domain wall (Fig. 4b) and the magnetization rotates slowly more perpendicular to the wave vector of propagating spin waves (Fig. 1a). Both effects change the spin-wave dispersion relation. While a decrease of effective field shifts the dispersion curve down, magnetization rotation towards the Damon-Eshbach configuration increases its slope and, thereby, the spin-wave group velocity. The larger group velocity within the head-to-head domain wall reduces the decay of propagating spin waves. Consequently, the spin-wave amplitude after passing the domain wall is somewhat larger compared to its value in a single domain. This effect is illustrated by the dashed line in Fig. 4a, showing the envelope function of propagating spin waves if no domain wall is present. Local changes in the dispersion relation also modify the spin-wave wavelength. Tuning of the group velocity and wavelength inside the head-to-head domain wall produces a finite phase shift upon spin-wave transmission, as illustrated by a change of slope in the contour plot of Fig. $4 \mathrm{c}$.

In contrast, spin waves are strongly reflected by the narrow $90^{\circ}$ head-to-tail domain wall (Fig. 4d, f). The effective magnetic field inside this domain wall peaks sharply at its center (Fig. 4e). Two field minima at $x= \pm 20 \mathrm{~nm}$ surround this peak. The non-uniform field profile produces a resonance mode that is characterized by two oscillatory out-of-phase antinodes on opposite sides of the domain-wall center (see inset in Fig. 4f and Supplementary movie 1). Propagating spin waves are absorbed and reflected by this domain-wall resonance mode, as illustrated by the undulations of spin-wave maxima and minima in the contour graph of Fig. 4f. The undulations are produced by interference of counter- 
propagating spin waves. Because of this interference effect, the spin-wave amplitude in front of the head-to-tail domain wall (Fig. 4d) is reduced in comparison to the head-to-head configuration (Fig. 4a), in agreement with the experimental data of Fig. 3a, d. Similar resonance modes have been simulated previously for $180^{\circ}$ domain walls in magnetic nanowires, and it was shown that oscillating antinodes can trigger spin-wave emission ${ }^{40}$ or cause resonant reflection of incoming spin waves ${ }^{22}$.

Figure 5 shows the frequency dependence of the spin-wave filtering effect. In panel $b$, we plot the amplitude of spin waves after passing a magnetic domain wall, relative to their amplitude in the same film without domain wall. Obviously, the $90^{\circ}$ headto-tail wall reduces the spin-wave amplitude in the frequency range from 9 to $13 \mathrm{GHz}$, with almost zero transmission around $11 \mathrm{GHz}$. This simulation result agrees well with the experimental data in Fig. 3. Compared to a single domain, a local enhancement of the group velocity inside the broad head-to-head domain wall reduces the attenuation of spin waves. The frequency dependence of the $m_{z, \mathrm{DW}} / m_{\mathrm{z}, \mathrm{NoDW}}$ ratio for this domain wall is qualitatively explained by the variation of $m_{\mathrm{z}}$ with group velocity $\left(v_{\mathrm{g}}\right)$ and spin-wave scattering time $(\tau), m_{\mathrm{z}}(x) \propto \exp \left(-x / v_{\mathrm{g}} \tau\right)$, where $v_{\mathrm{g}} \tau$ corresponds to the spin-wave decay length $\left(l_{\mathrm{d}}\right)$. Since the spinwave scattering time is approximated by $\tau=1 / 2 \pi \alpha f^{41}$, we can write $m_{\mathrm{z}, \mathrm{DW}} / m_{\mathrm{z}, \mathrm{NoDW}} \propto \exp \left(2 \pi x \alpha f\left(1 / v_{\mathrm{g}, \mathrm{NoDW}}-1 / v_{\mathrm{g}, \mathrm{DW}}\right)\right)$. Because the spin-wave group velocity is enhanced inside the head-to-head domain wall $\left(v_{\mathrm{g}, \mathrm{DW}}>v_{\mathrm{g}, \mathrm{NoDW}}\right)$, this expression predicts an exponential increase of $m_{\mathrm{z}, \mathrm{DW}} / m_{\mathrm{z}, \mathrm{NoDW}}$ with frequency. We emphasize that Fig. 5b depicts the frequency evolution of the $m_{\mathrm{z}, \mathrm{DW}} / m_{\mathrm{z}, \mathrm{NoDW}}$ ratio rather than the amplitude of spin waves after passing the head-to-head domain wall $\left(m_{\mathrm{z}}\right.$, a

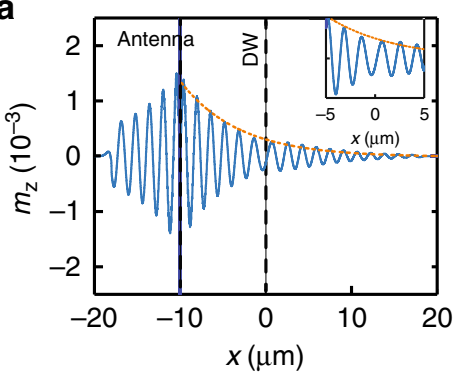

d

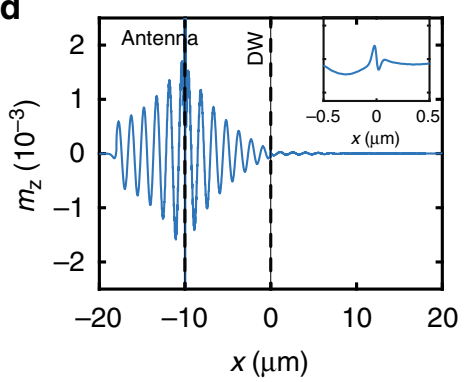

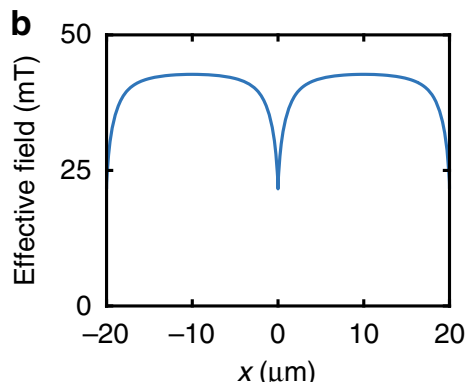
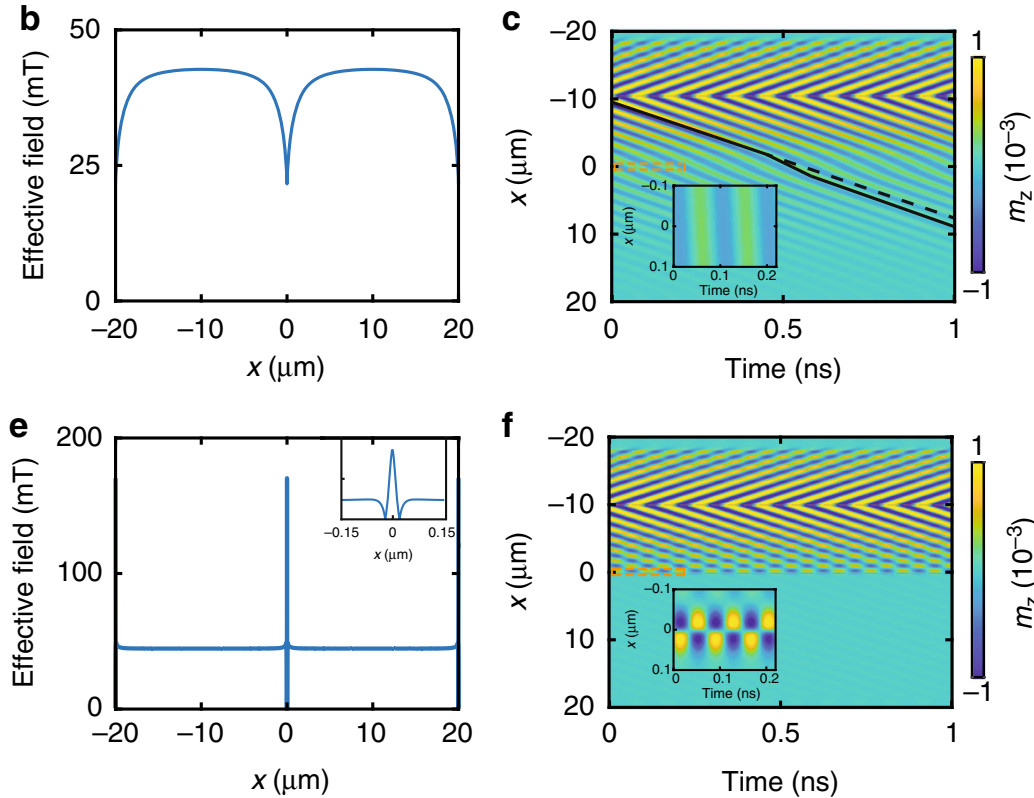

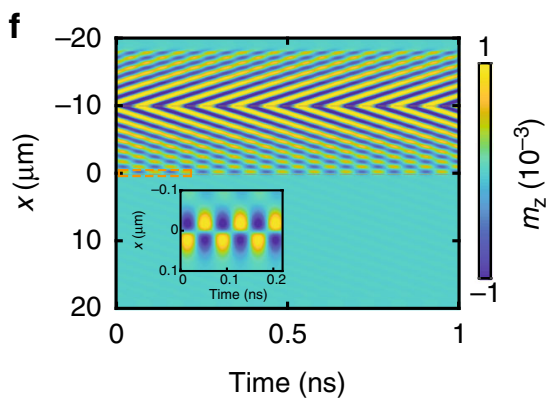

Fig. 4 Micromagnetic simulations of spin-wave transmission through single domain walls. Spin-wave transport through a broad $90^{\circ}$ head-to-head domain wall $(\mathbf{a}, \mathbf{c})$ and a narrow $90^{\circ}$ head-to-tail domain wall $(\mathbf{d}, \mathbf{f})$ at a frequency of $10.85 \mathrm{GHz}$. The panels show data for the steady excitation state, which is reached 9 ns after the sinusoidal magnetic field is turned on. The effective magnetic fields of the two domain walls are plotted in $\mathbf{b}$ and $\mathbf{e}$. The dashed line in a depicts the amplitude of propagating spin waves if no domain wall is present. The inset in $\mathbf{f}$ illustrates strong out-of-phase oscillations of two anti-nodes inside the head-to-tail domain wall

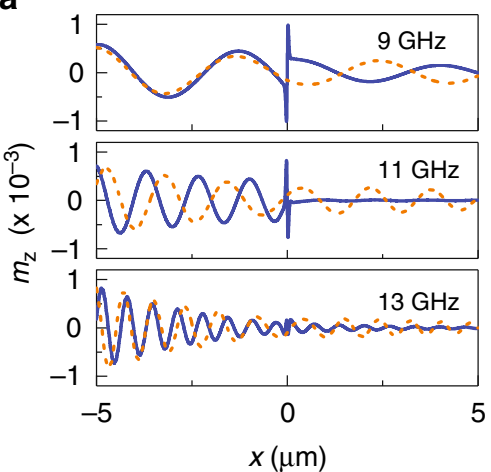

b

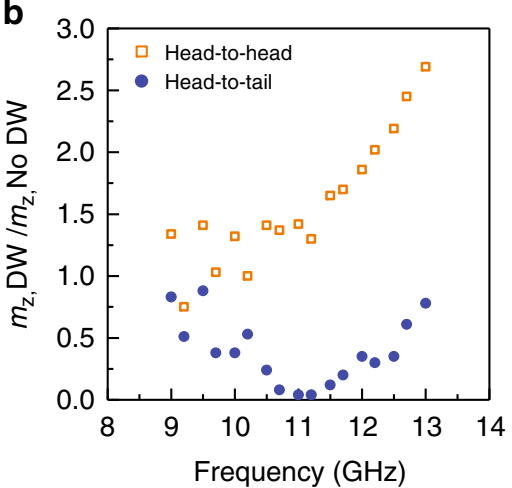

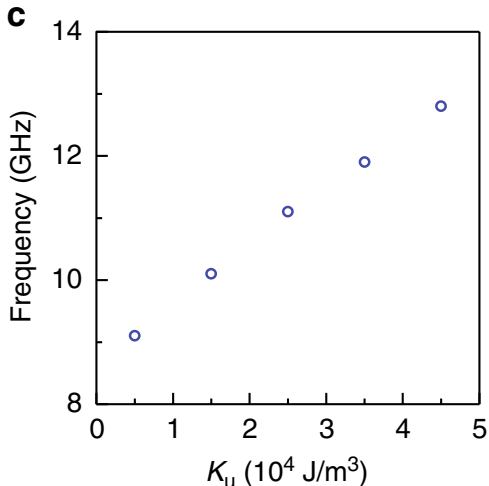

Fig. 5 Dependence of spin-wave transmission on frequency and anisotropy strength. a Micromagnetic simulations of spin-wave transport through a broad $90^{\circ}$ head-to-head domain wall (dashed orange line) and a narrow $90^{\circ}$ head-to-tail domain wall (solid blue line) at three different frequencies. $\mathbf{b}$ Frequency dependence of the spin-wave amplitude after passing through a head-to-head and head-to-tail domain wall. The amplitude is normalized to simulation data for a film without a domain wall. c Frequency of minimal spin-wave transmission through a head-to-tail domain wall as a function of magnetic anisotropy strength 
Dw). The amplitude of spin waves decreases with frequency because of an overall reduction of the spin-wave decay length, as illustrated by a comparison of the dashed orange curves in Fig. 5a.

Magnetic spin-wave valve. Following our results on spin-wave transmission through single $90^{\circ}$ domain walls, we propose a new structure for active spin-wave manipulation. Our device concept consists of three stripe domains with uniaxial magnetic anisotropy and two pinned domain walls. In this configuration, magnetization reversal in the central domain switches the domainwall state between a head-to-head/tail-to-tail combination and two head-to-tail walls (Fig. 1c). In practice, toggling between these two remanent magnetization states can be achieved by applying a magnetic-field pulse along the anisotropy axis of the central domain. Switching broad domain walls into narrow domain walls dramatically changes the transmission of spin waves near the domain-wall resonance frequency. An example at $f=11 \mathrm{GHz}$ is shown in Fig. 6. The pinned domain walls are separated by $1.5 \mu \mathrm{m}$ in this simulation. For the narrow head-totail walls, this distance is sufficient to reach a spin rotation of $90^{\circ}$ (Fig. 6b). In contrast, the spin rotation is only $38^{\circ}$ for the structure with a head-to-head/tail-to-tail wall combination. Irrespective of this finite-size scaling effect ${ }^{36}$, the configuration with broad domain walls is fully transparent for propagating spin waves (Fig. 6a, c). Excitation of a resonance mode in the narrow domain walls, on the other hand, reduces spin-wave transmission to nearly $0 \%$ (Fig. 6d, f). Since the spin-wave signal can be easily turned on or off by magnetic switching of the central domain, we refer to this structure as a magnetic spin-wave valve ${ }^{42}$.

\section{Discussion}

Just like reconfigurable magnonic crystals ${ }^{11,12,15}$, properties of pinned $90^{\circ}$ domain walls can be changed on demand, enabling their application in magnon conduits, filters, or logic gates. Moreover, since narrow and broad domain-wall states are nonvolatile, they can also be used to store data. Downscaling of programmable domain-wall filters is limited by the head-to-tail domain wall size. For typical materials and uniaxial anisotropy strengths, the width of this domain wall is several tens of nanometers. In nanoscale elements, broad head-to-head/tail-to-tail domain walls cannot form and, consequently, the magnetization of this state is almost uniform ${ }^{36}$. Spin waves propagate through this magnetization configuration without significant pertubation, enabling deterministic switching between a transparent state without domain walls and a strongly reflective state with a narrow head-to-tail wall. To realize switching in patterned devices, one could use local Oersted fields from a metallic nanowire that is placed on top of the ferromagnetic film.

Field control over the width of pinned magnetic domain walls requires an abrupt change of magnetic anisotropy. Here, we used strain coupling in a $\mathrm{CoFeB} / \mathrm{BaTiO}_{3}$ bilayer to demonstrate programmable spin-wave filtering. There are, however, other means by which lateral modulations of magnetic anisotropy can be realized. Examples include area-selective ion irradiation ${ }^{43,44}$ and thermally assisted scanning probe lithography ${ }^{45}$. Fabrication of pinned domain walls for active spin-wave manipulation is thus not limited to the use of specific substrates.

For the experimental parameters of our system, the transmission of spin waves through head-to-tail domain walls reduces drastically by resonant reflection around $11 \mathrm{GHz}$. In analogy to magnonic crystals, we estimate a spin-wave bandgap from the transmission curve (Fig. 5b). Using the full width at half maximum, we find a bandgap $\Delta f \approx 2 \mathrm{GHz}$. The domain-wall resonance mode and, thereby, the operation frequency of the spinwave filter can be tuned by varying the anisotropy strength (Fig. 5c). Lowering of the uniaxial magnetic anisotropy reduces the frequency of minimal spin-wave transmission. In our straincoupled system, this could be achieved by a change of the deposition parameters, the insertion of a seed layer at the ferromagnetic/ferroelectric interface, or the use of a ferromagnetic material with a smaller magnetostriction constant.

In summary, we report on active spin-wave manipulation using programmable domain walls. Control over the transmission of
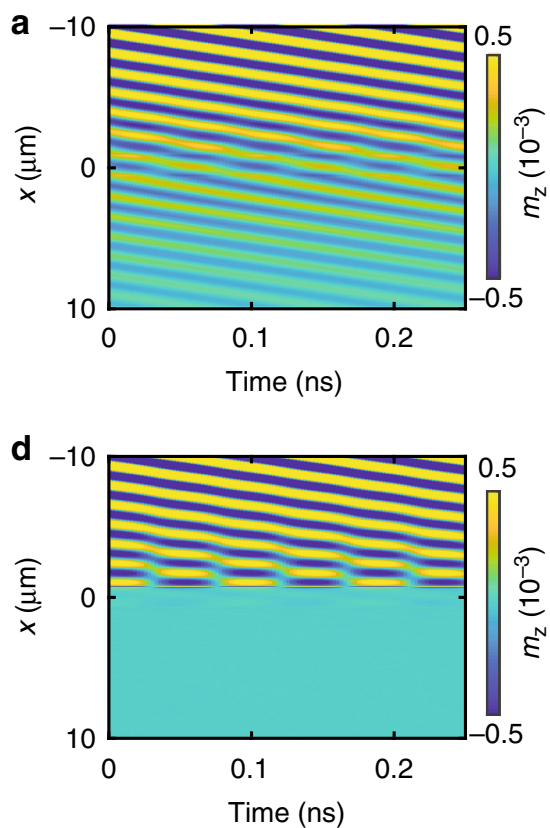
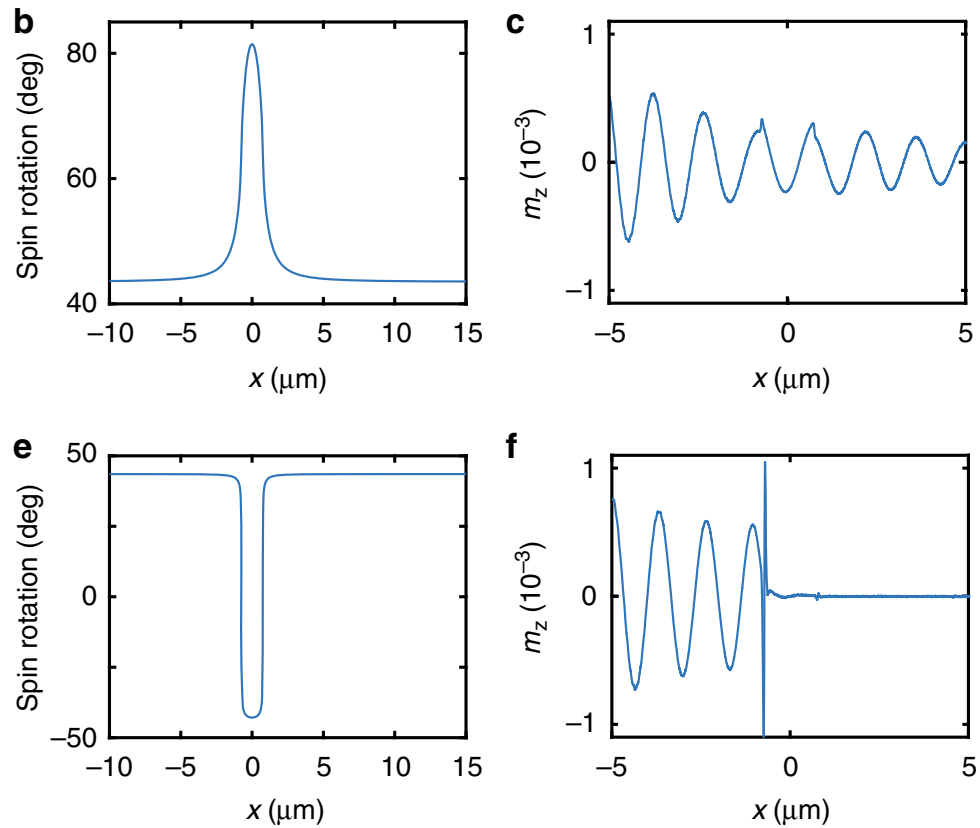

Fig. 6 Micromagnetic simulations of a magnetic spin-wave valve. In the simulations, the distance between two pinned domain walls is $1.5 \mu \mathrm{m}$ and the frequency is $11 \mathrm{GHz}$. Spin waves are excited at $x=-10 \mu \mathrm{m}$, and the panels show data for steady-state excitation. Magnetization reversal in the central domain transforms a head-to-head/tail-to-tail wall combination (transport characteristics in $\mathbf{a}, \mathbf{c}$ ) into two head-to-tail domain walls (transport results in $\mathbf{d}$, $\mathbf{f})$, or vice versa. Spin rotations between domains for these two states are shown in $\mathbf{b}$ and $\mathbf{e}$ 
propagating spin waves is realized by pinning domain walls at $90^{\circ}$ anisotropy boundaries. If the domain wall is set to a head-to-head or tail-to-tail configuration, it is broad and transmits propagating spin waves efficiently. Switching to a narrow head-to-tail domain wall reduces the transmission of spin waves to nearly $0 \%$ at the domain-wall resonance frequency. Both magnetization configurations are non-volatile and toggling between the two domainwall states is fully reversible and easily attained by magnetic switching in one of the domains.

\section{Methods}

Sample preparation. We grew the 50-nm-thick $\mathrm{CoFeB}$ film with a composition of $40 \% \mathrm{Co}, 40 \% \mathrm{Fe}$, and $20 \% \mathrm{~B}$ on a single-crystal $\mathrm{BaTiO}_{3}$ substrate using dc magnetron sputtering at $175^{\circ} \mathrm{C}$. At this temperature, $\mathrm{BaTiO}_{3}$ is paraelectric, and its lattice exhibits cubic symmetry. During post-deposition cooling through the paraelectric-to-ferroelectric phase transition at $120^{\circ} \mathrm{C}$, the structure of $\mathrm{BaTiO}_{3}$ becomes tetragonal and a regular pattern of ferroelectric stripe domains forms. The polarization in the domains is oriented in-plane and, together with the elongated axis of the tetragonal unit cell, it rotates by $90^{\circ}$ at domain boundaries. The phase transition in the $\mathrm{BaTiO}_{3}$ substrate strains the CoFeB film. Via inverse magnetostriction, this strain induces regular rotations of the uniaxial magnetic anisotropy axis. Since domain walls are strongly pinned by the magnetic anisotropy boundaries, it is possible to control their spin structure by the application of a magnetic field ${ }^{35}$. After cooling to room temperature, we covered the CoFeB film with a $3 \mathrm{~nm}$ $\mathrm{Ta} / 28 \mathrm{~nm} \mathrm{TaO}_{x}$ bilayer. The $\mathrm{TaO}_{x}$ film was grown by reactive sputtering. Microwave antennas with a width of $500 \mathrm{~nm}$ were patterned onto the $\mathrm{TaO}_{x}$ film using electron-beam lithography and lift-off. The broadband antennas consisted of $3 \mathrm{~nm}$ $\mathrm{Ta}$ and $50 \mathrm{~nm} \mathrm{Au}$

\section{Magnetic characterization and Brillouin light scattering experiments. The} magnetic domain structure of the sample was imaged using a wide-field magnetooptical Kerr microscope with $\times 20$ and $\times 100$ objectives. We used vibrating sample magnetometry to measure the saturation magnetization of the CoFeB film. From vector network analyzer ferromagnetic resonance (VNA-FMR) measurements, we derived a magnetic damping parameter of 0.007 . $\mu$-BLS measurements, with and without phase resolution, were performed by scanning a laser beam spot with a diameter of about $235 \mathrm{~nm}^{46}$ near the center of an excitation antenna and along the direction of propagating spin waves. The antenna was connected via rf picoprobes to a microwave generator working up to $20 \mathrm{GHz}$. We selected an antenna with a pinned domain wall in its vicinity. To maintain positional accuracy, we supplied the same reference image as feedback to image recognition-based drift stabilization software in experiments on both head-to-head and head-to-tail domain walls. More details on the $\mu$-BLS setup can be found in ref. ${ }^{47}$.

Micromagnetic simulations. We performed micromagnetic simulations using open-source GPU-accelerated MuMax3 software. Before spin-wave excitation, the simulation geometry was initialized by aligning the magnetization along the uniaxial anisotropy axes and letting the system reach its ground state in zero magnetic field. The domain-wall widths were calculated for this ground state using the same procedure as in Refs. ${ }^{35}$ and ${ }^{36}$. Previous work on high-resolution experimental characterization of pinned $90^{\circ}$ magnetic domain walls has shown that micromagnetic simulations provide a good estimate of their width ${ }^{37,38}$. After selection of the domain-wall type, spin waves were excited locally by a $100 \mathrm{mT}$ out-of-plane sinusoidal magnetic field. The ac field was applied to a one-cell-wide line at the center of one of the domains. To visualize propagating spin waves, we recorded the $z$-component of magnetization after reaching steady-state excitation. It took about $9 \mathrm{~ns}$ to reach this state. Besides information on propagating spin waves, the simulation signal also contained a dc component caused by $\sim 2^{\circ}$ out-of-plane magnetization tilting inside the domain walls. For clarity, we subtracted this dc component from the simulation data.

\section{Data availability}

The data that support the findings of this study are available from the corresponding author upon request.

Received: 9 May 2018 Accepted: 25 October 2018

Published online: 19 November 2018

\section{References}

1. Chumak, A. V., Vasyuchka, V. I., Serga, A. A. \& Hillebrands, B. Magnon spintronics. Nat. Phys. 11, 453-461 (2015).

2. $\mathrm{Yu}, \mathrm{H}$. et al. High propagating velocity of spin waves and temperature dependent damping in a CoFeB thin film. Appl. Phys. Lett. 100, 262412 (2012).
3. Liu, C. et al. Long-distance propagation of short-wavelength spin waves. Nat. Commun. 9, 738 (2018).

4. $\mathrm{Yu}, \mathrm{H}$. et al. Approaching soft X-ray wavelengths in nanomagnet-based microwave technology. Nat. Commun. 7, 11255 (2016).

5. van de Wiele, B., Hämäläinen, S. J., Baláž, P., Montoncello, F. \& van Dijken, S. Tunable short-wavelength spin wave excitation from pinned magnetic domain walls. Sci. Rep. 6, 21330 (2016).

6. Stigloher, J. et al. Spin-wave wavelength down-conversion at thickness steps. Appl. Phys. Express 11, 053002 (2018).

7. Schneider, T. et al. Realization of spin-wave logic gates. Appl. Phys. Lett. 92, 022505 (2008).

8. Lee, K.-S. \& Kim, S.-K. Conceptual design of spin wave logic gates based on a Mach-Zehnder-type spin wave interferometer for universal logic functions. J. Appl. Phys. 104, 053909 (2008).

9. Khitun, A., Bao, M. \& Wang, K. L. Magnonic logic circuits. J. Phys. D. Appl. Phys. 43, 264005 (2010).

10. Chumak, A. V., Serga, A. A. \& Hillebrands, B. Magnon transistor for allmagnon data processing. Nat. Commun. 5, 4700 (2014).

11. Krawczyk, M. \& Grundler, D. Review and prospects of magnonic crystals and devices with reprogrammable band structure. J. Phys. Condens. Matter 26, 123202 (2014)

12. Chumak, A. V., Serga, A. A. \& Hillebrands, B. Magnonic crystals for data processing. J. Phys. D. Appl. Phys. 50, 244001 (2017).

13. Chumak, A. V., Neumann, T., Serga, A. A., Hillebrands, B. \& Kostylev, M. P. A current-controlled, dynamic magnonic crystal. J. Phys. D. Appl. Phys. 42, 205005 (2009).

14. Vogel, M. et al. Optically reconfigurable magnetic materials. Nat. Phys. 11, 487-491 (2015).

15. Topp, J., Heitmann, D., Kostylev, M. P. \& Grundler, D. Making a reconfigurable artificial crystal by ordering bistable magnetic nanowires. Phys. Rev. Lett. 104, 207205 (2010).

16. Gubbiotti, G., Zhou, X., Haghshenasfard, Z., Cottam, M. G. \& Adeyeye, A. O. Reprogrammable magnonic band structure of layered permalloy/Cu/ permalloy nanowires. Phys. Rev. B 97, 134428 (2018).

17. Hertel, R., Wulfhekel, W. \& Kirschner, J. Domain-wall induced phase shifts in spin waves. Phys. Rev. Lett. 93, 257202 (2004).

18. Buijnsters, F. J., Ferreiros, Y., Fasolino, A. \& Katsnelson, M. I. Chiralitydependent transmission of spin waves through domain walls. Phys. Rev. Lett. 116, 147204 (2016).

19. Bayer, C., Schultheiss, H., Hillebrands, B. \& Stamps, R. L. Phase shift of spin waves traveling through a $180^{\circ}$ Bloch domain wall. IEEE Trans. Magn. 41, 3094-3096 (2005).

20. Chang, L.-J. et al. Ferromagnetic domain walls as spin wave filters and the interplay between domain walls and spin waves. Sci. Rep. 8, 3910 (2018).

21. Macke, S. \& Goll, D. Transmission and reflection of spin waves in the presence of NÃ®el walls. J. Phys. Conf. Ser. 200, 042015 (2010).

22. Wang, X.-G., Guo, G.-H., Zhang, G.-F., Nie, Y.-Z. \& Xia, Q.-L. An analytical approach to the interaction of a propagating spin wave and a Bloch wall. Appl. Phys. Lett. 102, 132401 (2013).

23. Han, D.-S. et al. Magnetic domain-wall motion by propagating spin waves. Appl. Phys. Lett. 94, 112502 (2009).

24. Kim, J.-S. et al. Interaction between propagating spin waves and domain walls on a ferromagnetic nanowire. Phys. Rev. B 85, 174428 (2012).

25. Wang, X.-G., Guo, G.-H., Nie, Y.-Z., Zhang, G.-F. \& Li, Z.-X. Domain wall motion induced by the magnonic spin current. Phys. Rev. B 86, 054445 (2012).

26. Wang, X.-G., Guo, G.-H., Zhang, G.-F., Nie, Y.-Z. \& Xia, Q.-L. Spin-wave resonance reflection and spin-wave induced domain wall displacement. $J$. Appl. Phys. 113, 213904 (2013).

27. Hinzke, D. \& Nowak, U. Domain wall motion by the magnonic spin Seebeck effect. Phys. Rev. Lett. 107, 027205 (2011).

28. Yan, P., Wang, X. S. \& Wang, X. R. All-magnonic spin-transfer torque and domain wall propagation. Phys. Rev. Lett. 107, 177207 (2011).

29. Woo, S., Delaney, T. \& Beach, G. S. D. Magnetic domain wall depinning assisted by spin wave bursts. Nat. Phys. 13, 448-454 (2017).

30. Buess, M., Raabe, J., Perzlmaier, K., Back, C. H. \& Quitmann, C. Interaction of magnetostatic excitations with $90^{\circ}$ domain walls in micrometer-sized permalloy squares. Phys. Rev. B 74, 100404 (2006).

31. Pirro, P. et al. Experimental observation of the interaction of propagating spin waves with Néel domain walls in a Landau domain structure. Appl. Phys. Lett. 106, 232405 (2015).

32. Lahtinen, T. H. E., Tuomi, J. O. \& van Dijken, S. Pattern transfer and electric field-induced magnetic domain formation in multiferroic heterostructures. Adv. Mater. 23, 3187-3191 (2011)

33. Lahtinen, T. H. E., Franke, K. J. A. \& van Dijken, S. Electric-field control of magnetic domain wall motion and local magnetization reversal. Sci. Rep. 2, 258 (2012).

34. Hämäläinen, S. J., Brandl, F., Franke, K. J. A., Grundler, D. \& van Dijken, S. Tunable short-wavelength spin-wave emission and confinement in 
anisotropy-modulated multiferroic heterostructures. Phys. Rev. Appl. 8, 014020 (2017).

35. Franke, K. J. A., Lahtinen, T. H. E. \& van Dijken, S. Field tuning of ferromagnetic domain walls on elastically coupled ferroelectric domain boundaries. Phys. Rev. B 85, 094423 (2012).

36. Franke, K. J. A., López González, D., Hämäläinen, S. J. \& van Dijken, S. Size dependence of domain pattern transfer in multiferroic heterostructures. Phys. Rev. Lett. 112, 017201 (2014).

37. Casiraghi, A. et al. Influence of elastically pinned magnetic domain walls on magnetization reversal in multiferroic heterostructures. Phys. Rev. B 92, 054406 (2015).

38. López González, D., Casiraghi, A., Kronast, F., Franke, K. J. A. \& van Dijken, $\mathrm{S}$. Influence of magnetic field and ferromagnetic film thickness on domain pattern transfer in multiferroic heterostructures. J. Magn. Magn. Mater. 441, 404-408 (2017).

39. Vansteenkiste, A. et al. The design and verification of MuMax3. AIP Adv. 4 , 107133 (2014).

40. Hermsdoerfer, S. J. et al. A spin-wave frequency doubler by domain wall oscillation. Appl. Phys. Lett. 94, 223510 (2009).

41. Madami, M. et al. Direct observation of a propagating spin wave induced by spin-transfer torque. Nat. Nanotechnol. 6, 635-638 (2011).

42. Wu, H. et al. Magnon valve effect between two magnetic insulators. Phys. Rev. Lett. 120, 097205 (2018)

43. Trützschler, J. et al. Optimization of magneto-resistive response of ionirradiated exchange biased films through zigzag arrangement of magnetization. J. Appl. Phys. 115, 103901 (2014).

44. Trützschler, J., Sentosun, K., Mozooni, B., Mattheis, R. \& McCord, J. Magnetic domain wall gratings for magnetization reversal tuning and confined dynamic mode localization. Sci. Rep. 6, 30761 (2016).

45. Albisetti, E. et al. Nanopatterning reconfigurable magnetic landscapes via thermally assisted scanning probe lithography. Nat. Nanotechnol. 11, 545-551 (2016).

46. Gubbiotti, G. et al. Setup of a new Brillouin light scattering apparatus with submicrometric lateral resolution and its application to the study of spin modes in nanomagnets. J. Appl. Phys. 105, 07D521 (2009).

47. Madami, M., Gubbiotti, G., Tacchi, S. \& Carlotti, G. Application of microfocused Brillouin light scattering to the study of spin waves in lowdimensional magnetic systems. Solid State Phys. 63, 79-150 (2012).

\section{Acknowledgements}

This work was supported by the European Research Council (Grant Nos. ERC-2012-StG 307502-E-CONTROL and ERC-PoC-2018 812841-POWERSPIN) and the Academy of
Finland (Grant Nos. 317918 and 316857). S.J.H. acknowledges financial support from the Väisälä Foundation. Lithography was performed at the Micronova Nanofabrication Centre, supported by Aalto University. We also acknowledge the computational resources provided by the Aalto Science-IT project.

\section{Author contributions}

S.J.H. and S.v.D. designed and initiated the research. S.J.H. fabricated the samples. S.J.H. M.M. and G.G. conducted the $\mu$-BLS measurements. S.J.H. and H.J.Q. performed the micromagnetic simulations. S.v.D. supervised the project. S.J.H. and S.v.D. wrote the manuscript, with input from all other authors.

\section{Additional information}

Supplementary Information accompanies this paper at https://doi.org/10.1038/s41467018-07372-x.

Competing interests: The authors declare no competing interests.

Reprints and permission information is available online at http://npg.nature.com/ reprintsandpermissions/

Publisher's note: Springer Nature remains neutral with regard to jurisdictional claims in published maps and institutional affiliations.

\section{(c) (i)}

Open Access This article is licensed under a Creative Commons Attribution 4.0 International License, which permits use, sharing, adaptation, distribution and reproduction in any medium or format, as long as you give appropriate credit to the original author(s) and the source, provide a link to the Creative Commons license, and indicate if changes were made. The images or other third party material in this article are included in the article's Creative Commons license, unless indicated otherwise in a credit line to the material. If material is not included in the article's Creative Commons license and your intended use is not permitted by statutory regulation or exceeds the permitted use, you will need to obtain permission directly from the copyright holder. To view a copy of this license, visit http://creativecommons.org/ licenses/by/4.0/

(c) The Author(s) 2018 